\title{
Essentials of Specimen Preparation for High Performance Volume Imaging by SEM
}

\author{
Thomas J. Deerinck ${ }^{1}$
}

${ }^{1}$ The National Center for Microscopy and Imaging Research, UCSD, La Jolla, California, USA.

The unlikely application of scanning electron microscopy (SEM) to 3-D imaging of epoxy embedded specimens has burgeoned over the last few years and will likely eventually supplant conventional TEM as the mainstay of wide-field nanometer-scale cytology of biological specimens. Three different approaches have come to the forefront: Serial block-face SEM (SBEM), whereby a specialized miniature ultramicrotome is fitted into a SEM and the mirror-smooth block-face imaged using backscatter electrons (BSEs) in between automated removal of the surface using a diamond knife; focused ion beam SEM (FIB-SEM), which achieves much the same thing but instead removes the surface material using a focused ion beam; and array tomography SEM (AT-SEM), employing ribbons of serial sections prepared using an ultramicrotome that are mounted on special tape or silicon wafers prior to BSE-SEM imaging. Each of these methods has their own strengths and weaknesses (for review, see [1]), but common to each are the approaches used to prepare biological specimens prior to imaging. Since BSE yield is meager at the low accelerating voltages typically employed (1-4 keV), combinatorial intense heavy metal staining of cells and tissues is needed to maximize BSE yield and improve specimen conductivity in order to minimize specimen charging; important since the samples are embedded in nonconductive epoxy resins. One of the first staining protocols developed specifically to maximize BSE yield from epoxy embedded specimens by SEM employed a variety of previously introduced heavymetal stains, including potassium ferrocyanide-reduced osmium tetroxide, liganding with thiocarbohydrazide followed by a second osmium tetroxide treatment, and en bloc uranyl acetate and lead aspartate staining prior to epoxy embedding [2]. This approach made possible block-face imaging of tissues at high vacuum instead of variable-pressure SEM, vastly improving image acquisition speed and achievable resolution. Recently, a protocol (termed BROPA, for brain-wide reduced-osmium staining with pyrogallol-mediated amplification) was developed to achieve homogeneous staining of the entire mouse brain [3], and subsequent modifications have drastically shortened the time required to prepare samples using this method [4]. The ultimate goal of these and future approaches is to try and obtain the same type of image information using these SEM techniques as is currently obtained by ultrathin-section transmission EM (figure 1).

In addition to these protocols, new molecular-genetic imaging probes such as miniSOG [5] and the ascorbate peroxidase derivative APEX [6], as well as new chemical labels [7, 8] have been developed to selectively contrast macromolecules in 3-D with excellent cellular preservation, since they do not require compromises to primary chemical fixation or the use of permeablizing detergents, and are all easily adapted to SBEM, FIB-SEM and AT-SEM (figure 2). These probes and others under development promise to greatly improve the ability for 3-D localization of cellular constituents at high resolution. Furthermore, a method to combine hybrid high-pressure freezing methods with EM-level genetic labeling and heavy metal staining has been introduced, thereby enhancing subcellular morphological preservation of difficult to preserve samples [9]. Finally, the introduction of gasinjection based charge compensation has essentially eliminated specimen charging of samples using SBEM and allows for high vacuum imaging for vastly improved resolution from any sample [10]. 


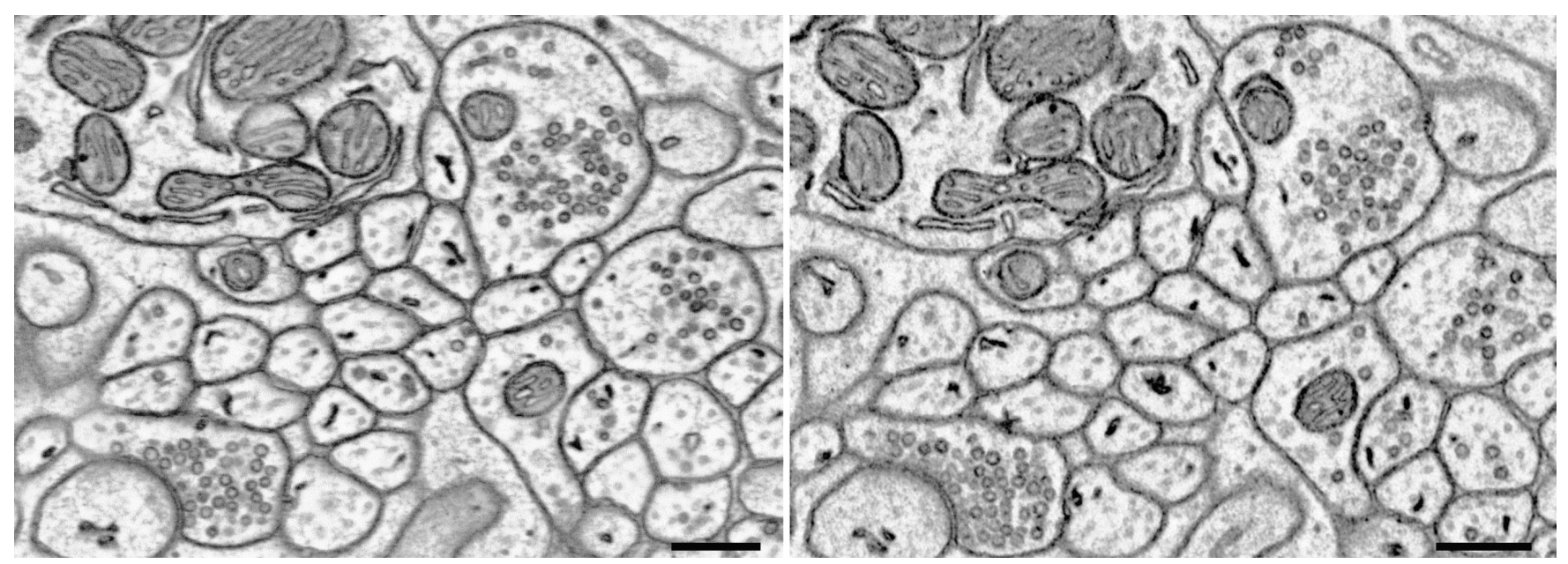

Figure 1. Block-face BSE-SEM image taken at $1.9 \mathrm{keV}$ (left panel) and the corresponding serial ultrathin-section TEM image of the same region taken at $80 \mathrm{keV}$ (right panel) using heavy metal stained brain tissue prepared as described [2]. The images are nearly equivalent. Bars $=0.25$ microns.
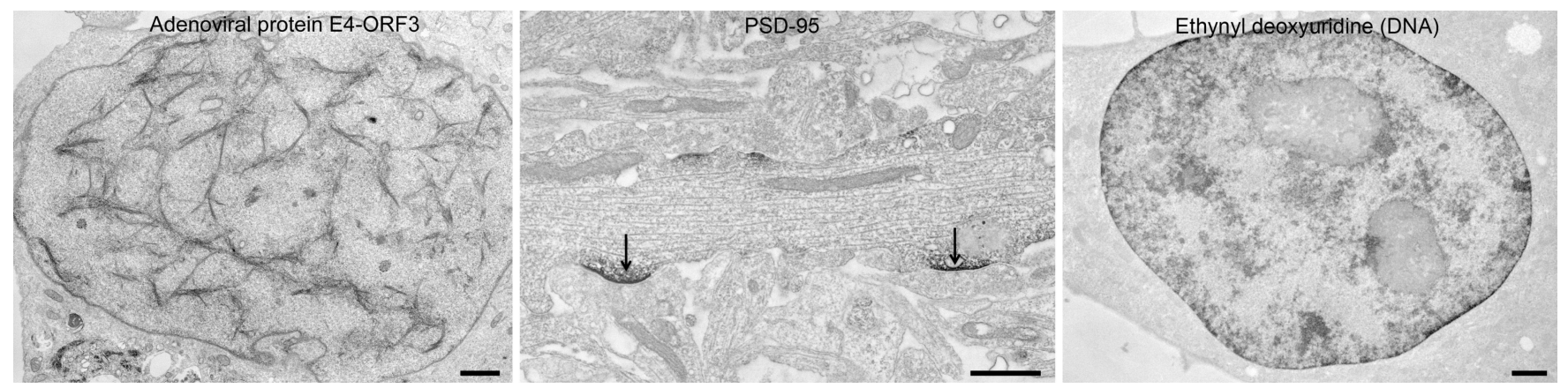

Figure 2. The genetic labeling probe miniSOG (left panel) used to localize the viral protein E4-ORF3 in a cultured cell nucleus, APEX2 used to label the protein PSD-95 (arrows) in cultured hippocampal neuron synapses (middle panel), and click-chemistry labeled DNA incorporated with 5-ethynyl-2'deoxyuridine (right panel). Bars $=0.5$ microns.

[1] B Tietze and C Genoud, Biol Cell 108, (2016), p. 307-323.

[2] T Deerinck, et al. Microsc. Microanal. 16 (2010), p. 1138-1139.

[3] S Mikula and W Denk. Nat. Methods 12 (2015), p. 541-546.

[4] C Genoud et al., Front. Neuroanat. 12 (2018), p. 1-8.

[5] X Shu et al. PLoS Biol. 9 (2011), p. 1-10.

[6] J Martell et al. Nat. Biotechnol. 30 (2012), p. 1143-1148

[7] J Ngo et al. Nat. Chem. Bio. 12 (2016), p. 459-465.

[8] H Ou et al. Science 357 (2017).

[9] T Tsang et al. eLIFE 7 (2018).

[10] T Deerinck et al. J. Microscopy 270, (2017), p. 142-149.

[11] Funding for this work was from NIGMS (P41GM103412) to M. Ellisman, NCMIR. 